\title{
État des lieux de la prise en charge de la douleur
}

\section{Acute Pain Management in the Emergency Setting: State of the Art and Perspectives}

\author{
A. Ricard-Hibon - A. Chareyron \\ Reçu le 10 octobre 2017; accepté le 13 janvier 2018 \\ (C) SFMU et Lavoisier SAS 2018
}

Résumé Le concept d'oligoanalgésie en structure d'urgences reste une réalité en France comme dans de nombreux pays qui publient sur le sujet. Les motifs de cette oligoanalgésie sont multiples, le plus souvent liés à des contraintes organisationnelles plus que médicales. Les solutions existent, et la douleur aiguë persistante ne doit plus être une fatalité en structure d'urgences. L'analyse des raisons de l'oligoanalgésie avec des audits ciblés et la mise en place de protocoles thérapeutiques locaux sont des prérequis à l'amélioration de la prise en charge. Les nouveaux enjeux de la médecine d'urgence, liés à l'augmentation constante de la sollicitation, mais également en lien avec l'évolution des techniques médicales et des compétences des équipes médicales et soignantes, donnent de nouvelles perspectives pour améliorer la qualité-sécurité de la prise en charge de la douleur en structure d'urgences.

Mots clés Douleur aiguë · Analgésie · Structure d'urgences · Oligoanalgésie · Qualité

\begin{abstract}
The oligo-analgesia in the emergency department (ED) is still a reality in France - such as in numbers on country studying acute pain in emergency care. Reasons for oligo-analgesia are well known, most often due to organisational challenge rather than medical barrier. Number of solutions exists to improve quality of analgesia in the ED, so persistent acute pain at discharge does not remain a fatality. The analysis of the reasons for oligo-analgesia with targeted audits and the implementation of local therapeutics protocols are the first step to improve analgesia in the ED. New challenges in emergency care due to increase in activity and also new medical techniques and skills in emergency care give
\end{abstract}

\footnotetext{
A. Ricard-Hibon $(\varangle) \cdot$ A. Chareyron

Services des urgences, SAMU, SMUR de Pontoise et Beaumont/ Oise

Pôle MUR (médecine d'urgence et réanimation) centre hospitalier René Dubos

6, avenue de l'Île-de-France, F-95300 Pontoise Cedex, France e-mail : agnes.ricard-hibon@ght-novo.fr
}

new perspectives to improve quality and security care for acute pain management in the ED.

Keywords Acute pain · Analgesia $\cdot$ Emergency care Oligo-analgesia · Quality

La douleur est un motif majeur de recours aux soins en urgence aussi bien en extra- qu'en intrahospitalier. La prise en charge de ces douleurs aiguës fait partie des préoccupations majeures des soignants au quotidien. En effet, le soulagement de ces patients est un objectif éthique et déontologique constant dans notre profession.

En dehors de la nécessité éthique de soulager la douleur pour assurer le confort du patient, l'analgésie facilite la prise en charge médicale sans compromettre la démarche diagnostique, contrairement à certaines idées reçues. La médicalisation des structures d'urgences autorise l'utilisation de médicaments et de techniques efficaces, lorsque les conditions de sécurité sont parfaitement respectées. Les nombreuses recommandations de bonnes pratiques, aussi bien nationales qu'internationales, visent à améliorer la prise en charge des structures d'urgences [1]. Malgré ces recommandations régulièrement actualisées, la douleur en situation d'urgence reste insuffisamment prise en considération. Plusieurs travaux rapportent un nombre insuffisant de patients traités et soulagés à l'issue de leur prise en charge en structure d'urgences $[2,3]$.

La Société française de médecine d'urgence - en lien avec d'autres sociétés savantes - a publié et actualisé des recommandations sur la prise en charge de la douleur en situation d'urgence [1], avec notamment l'organisation récente des Journées thématiques interactives de la SFMU à Grenoble sur l'analgésie-sédation. Au cours de ces Journées, les échanges professionnels des experts de l'analgésie ont permis de publier un référentiel scientifique, riche en données et en recommandations fondées sur l'actualisation récente de données de la littérature. Ce référentiel a pour but d'améliorer les prises en charge dans nos structures d'urgences [4]. 


\section{Épidémiologie de la douleur en structure d'urgences}

La douleur est un symptôme fréquent de recours dans nos structures d'urgences [2]. Selon les études, plus de $60 \%$ des patients expriment une douleur à l'admission aux urgences et près de $75 \%$ en extrahospitalier [5]. En Europe, le nombre annuel de patients, exprimant une douleur aux urgences, représente 11,8 millions de personnes en France, 13,6 millions en Italie et jusqu'à 15,4 millions de patients en Angleterre. La prise en charge de ces douleurs est un enjeu médical, paramédical et organisationnel pour une amélioration de la qualité des soins. En cas d'échec de soulagement des patients, l'impact sur la morbimortalité est important. En effet, la douleur a des effets physiopathologiques délétères sur l'organisme - aussi bien à court terme qu'à long terme - nécessitant une prise en charge précoce et efficace [1]. Cependant, malgré les recommandations nationales et internationales, le concept d'oligoanalgésie en structure d'urgences - décrit par Wilson et Pendleton en 1989 [6] - reste toujours d'actualité. Cependant, plusieurs études nationales et internationales décrivent des marges de progression dans ce domaine [2,7-8].

La SFMU a lancé une grande étude nationale en partenariat avec l'Agence nationale d'appui à la performance (ANAP), dans le cadre d'un projet national d'amélioration de la qualité de prise en charge de la douleur en structure d'urgences [2]. Cette étude a rassemblé 50 services d'urgences volontaires en France permettant d'inclure plus de 11000 patients, dont plus de 7200 douloureux. La douleur était jugée modérée à sévère chez $66 \%$ des patients inclus. Seuls $51 \%$ des patients douloureux avaient reçu un traitement antalgique aux urgences. Parmi les patients ayant eu une évaluation de l'intensité douloureuse à la sortie des urgences (seulement un patient sur deux est évalué), $27 \%$ exprimaient une douleur modérée à sévère à l'issue de leur prise en charge aux urgences. Ces résultats s'ajoutent à ceux publiés dans la littérature internationale et démontrent une problématique partagée par de nombreux soignants, quel que soit le pays d'origine $[7,8]$. En médecine d'urgence préhospitalière, cette oligoanalgésie est également observée. Dans l'étude de Bounes et al., 66 à $76 \%$ des patients étaient soulagés [9], et 53 à $73 \%$ dans l'étude de Ricard-Hibon et al. [10], malgré l'administration de morphiniques dès le début de la prise en charge. Dans une étude préhospitalière plus récente en Suisse, $43 \%$ des patients ne recevaient aucun antalgique [5].

\section{Raisons de l'oligoanalgésie dans les structures d'urgences}

L'amélioration des prises en charge passe par l'étape préalable d'identification des freins à l'administration d'antalgi- ques aux urgences. Les raisons de l'oligoanalgésie, décrites dans la littérature internationale, sont multiples, avec des particularités propres à différents pays. Certaines causes sont constamment retrouvées, quel que soit le pays d'origine : la sous-estimation de l'intensité douloureuse par le personnel médical et soignant, l'absence d'évaluation de l'intensité douloureuse à l'admission, la priorité donnée à l'urgence vitale, l'over-crowding des urgences, la notion de fatalité de la douleur en urgence, le manque de formation des professionnels, l'absence d'antalgiques puissants à disposition des soignants, la peur des effets secondaires liés aux morphiniques, les problèmes organisationnels des structures d'urgences, les dogmes et les mauvaises habitudes... [1]. L'oligoanalgésie des personnes âgées, par exemple, est constamment retrouvée dans la littérature, alors que certaines études - notamment postopératoires — témoignent de l'intérêt majeur d'une analgésie efficace dans cette population, sans majoration des effets secondaires [11-13]. La pathologie est également un facteur identifié d'oligoanalgésie, dans l'analyse multicentrique française. En effet, la traumatologie périphérique est insuffisamment prise en charge [2], la douleur abdominale insuffisamment traitée sous le prétexte de masquer un diagnostic ou une complication [14-17].

En revanche, d'autres étiologies ne sont pas universelles. Dans les pays anglo-saxons, les freins à l'analgésie préhospitalière sont essentiellement liés à des particularités réglementaires liées à l'absence de médicalisation préhospitalière. Cela n'est pas une problématique en France ni dans beaucoup de pays d'Europe. Dans le domaine des dogmes et des mauvaises habitudes, la littérature outre-Atlantique rapporte des disparités ethniques importantes, qui ne sont pas retrouvées (ou pas étudiées ?) en Europe [18]. De même, les disparités liées au sexe, avec une sous-utilisation des antalgiques, n'ont pas été retrouvées dans l'étude multicentrique française, contrairement à la littérature internationale : une spécificité française appréciable [2,19].

Enfin, la douleur induite par les soins est une réalité dans les structures d'urgences. De nombreux soins sont effectués tous les jours aux urgences, dont un certain nombre engendre ou aggrave des douleurs préexistantes, sans que celles-ci aient été anticipées ou prévenues par une antalgie adaptée. Dans l'étude de Singer et al., les gestes les plus courants aux urgences ont été évalués en termes d'intensité douloureuse par les patients et le personnel soignant en insu. Elle montre une nette disparité entre les évaluations effectuées par les soignantes versus celles évaluées par les patients [20]. Dans l'étude Paliers, seuls $25 \%$ des patients avaient reçu un antalgique pour le geste douloureux - majoritairement un antalgique de palier 1, quelle que soit l'intensité de la douleur -, et $47 \%$ des patients exprimaient une douleur toujours présente à la sortie des urgences [21].

Toutes ces causes identifiées ont des solutions préconisées, possibles à mettre en œuvre. En effet, la douleur aiguë 
n'est plus une fatalité en médecine d'urgence et peut être soulagée rapidement. En quelques années, l'amélioration est sensible, mais les marges de progression existent encore. La mobilisation de l'ensemble des personnels soignants médicaux et paramédicaux est nécessaire pour atteindre le but recherché : le soulagement de tous les patients avant leur sortie des structures d'urgences.

\section{Pistes d'amélioration accessibles à une mise en application à courte échéance}

L'amélioration de la prise en charge de la douleur dans les structures d'urgences passe, en premier lieu, par une sensibilisation du personnel à la problématique - en leur donnant leurs propres chiffres obtenus dans le service. Le meilleur moyen de sensibiliser les professionnels est de leur montrer ce qu'ils font au quotidien et ce qu'ils pourraient faire en termes de marge de progression. Toutes les démarches qualité ayant débuté par un audit ciblé impliquant le personnel soignant, puis suivies de mesures correctrices initiées par ces personnels impliqués, montrent une amélioration [22-24]. Le meilleur « moteur » de l'amélioration de la qualité au sein d'une équipe est de montrer l'efficacité de son investissement, et - dans le domaine de la douleur - l'implication du personnel paramédical est particulièrement efficace.

La mise en place de protocoles de traitement antalgiques standardisés - initiés dès le tri IAO - favorise l'amélioration du nombre de patients traités et permet une meilleure homogénéité des pratiques. Néanmoins, les protocoles IAO ne suffisent pas à améliorer le taux de patients soulagés, s'ils ne sont pas adaptés à l'intensité douloureuse. En effet, on observe dans les études un recours important aux antalgiques de niveau 1 , mais un très faible recours aux antalgiques puissants malgré le nombre important de patients exprimant une douleur intense, avec un taux d'efficacité faible en termes de soulagement [2]. L'indicateur à surveiller n'est pas le nombre de patients ayant reçu un antalgique, mais plutôt le nombre de patients ayant reçu un antalgique adapté à l'intensité douloureuse exprimée. Le recours à l'analgésie multimodale est particulièrement adapté à la douleur aiguë en médecine d'urgence - associant l'analgésie inhalée, l'analgésie locorégionale, l'analgésie orale utilisant les paliers 1 et 2 , mais également l'utilisation en première intention des morphiniques intraveineux pour les douleurs intenses en mono- ou multithérapie (association morphine-kétamine-MEOPA par exemple). Par ailleurs, les nouvelles modalités d'administration d'antalgiques, tels que la voie inhalée, sublinguale, transcutanée, autoadministration... sont des modalités à étudier pour s'adapter aux contraintes organisationnelles et à la gestion de flux des patients aux urgences : tels sont les nouveaux enjeux et perspectives pour améliorer encore et toujours le soulagement de la douleur aiguë en situation d'urgence.
Enfin, la sédation procédurale doit se développer dans les structures d'urgences pour tous les gestes douloureux faits en structure d'urgences. La littérature internationale montre l'intérêt grandissant des techniques de sédation procédurale utilisant notamment le propofol ou kétofol dans un rapport bénéfice/risque adapté - sous réserve de conditions de sécurité respectées $[4,24]$.

\section{Conclusion}

L'oligoanalgésie n'est pas une fatalité en structure d'urgences. Dans le principe du primum non nocere s'inscrit aussi la nécessité de ne pas laisser souffrir un patient en structure d'urgences. L'évolution des techniques médicales et des compétences des équipes donne de nouvelles perspectives pour améliorer la qualité-sécurité de la prise en charge de la douleur en structure d'urgences.

\section{Références}

1. Ricard-Hibon A, Bounes V (2012) Groupe d'experts SfarSFMU. Sédation-analgésie en médecine d'urgence : quelle analgésie-sédation du patient en ventilation spontanée ? Ann $\mathrm{Fr}$ Anesth Reanim 31:295-312

2. Guéant S, Taleb A, Borel-Kuhner J, et al (2010) Quality of pain management in the ED: results of a multicenter prospective study. Eur J Anaesthesiol 28:97-105

3. Lvovschi V, Aubrun F, Bonnet P, et al (2008) Intravenous morphine titration to treat severe pain in the ED. Am J Emerg Med 26:676-82

4. Desmettre T (2017) Journées thématiques interactives de la SFMU. Analgésie et sédation : références en médecine d'urgence. In: SFMU (ed), Paris, Lavoisier

5. Eidenbenz D, Taffé P, Hugli O, et al (2016) A two-year retrospective review of the determinants of pre-hospital analgesia administration by alpine helicopter emergency medical physicians to patients with isolated limb injury. Anaesthesia 71:779-8

6. Wilson J, Pendleton J (1989) Oligoanalgesia in the emergency department. Am J Emerg Med 7:620-3

7. Decosterd I, Hugli O, Tamches E, et al (2007) Oligoanalgesia in the emergency department: short-term beneficial effects of an education program on acute pain. Ann Emerg Med 50:462-71

8. Todd KH, Ducharme J, Choiniere M, et al (2007) Pain in the emergency department: results of the Pain and Emergency Medicine Initiative (PEMI) multicenter study. J Pain 8:460-6

9. Bounes V, Charpentier S, Houze-Cerfon $\mathrm{CH}$, et al (2008) Is there an ideal morphine dose for prehospital treatment of severe acute pain? A randomized, double-blind comparison of 2 doses. Am J Emerg Med 26:148-54

10. Ricard-Hibon A, Belpomme V, Chollet C, et al (2008) Compliance with morphine administration protocol and effect on pain relief in out-of-hospital patients. J Emerg Med 34:305-10

11. Keita H, Tubach F, Maalouli J, et al (2008) Age-adapted morphine titration produces equivalent analgesia and adverse effects in younger and older patients. Eur J Anaesthesiol 25:352-6

12. Aubrun F, Monsel S, Langeron O, et al (2002) Postoperative titration of intravenous morphine in the elderly patient. Anesthesiology 96:17-23 
13. Ricard-Hibon A, Duchateau FX, Vivien B (2012) Out-of-hospital management of elderly patients for trauma injury. Ann Fr Anesth Reanim 31:e7-e10

14. Vermeulen B, Morabia A, Unger PF, et al (1999) Acute appendicitis: influence of early pain relief on accuracy of clinical and US findings in the decision to operate - a randomized trial. Radiology 210:639-43

15. McHale P, LoVecchio F (2001) Narcotic analgesia in the acute abdomen - a review of prospective trials. Eur J Emerg Med 8:131-6

16. Manterola C, Astudillo P, Losada H, et al (2007) Analgesia in patients with acute abdominal pain. Cochrane Database Syst Rev 18:CD005660

17. Thomas SH, Silen W, Cheema F, et al (2003) Effects of morphine analgesia on diagnostic accuracy in emergency department patients with abdominal pain: a prospective, randomized trial. J Am Coll Surg 196:18-31

18. Todd KH, Deaton C, D'Adamo AP, Goe L (2000) Ethnicity and analgesic practice. Ann Emerg Med 35:11-6
19. Chen EH, Shofer FS, Dean AJ, et al (2008) Gender disparity in analgesic. treatment of emergency department patients with acute abdominal pain. Acad Emerg Med 15:414-8

20. Singer AJ, Richman PB, Kowalska A, et al (1999) Comparison of patient and practitioner assessments of pain from commonly performed emergency department procedures. Ann Emerg Med $33: 652-8$

21. Boccard E, Adnet F, Gueugniaud PY, et al (2011) Pain management in adult patients in emergency care units in France in 2010. Ann Fr Med Urg 1:312-9

22. Ricard-Hibon A, Ducasse JL, Ravaud P, et al (2004) Quality control programme for acute pain management in emergency medicine: a national survey. Eur J Emerg Med 11:198-203

23. Ricard-Hibon A, Chollet C, Saada S, et al (1999) A quality control program for acute pain management in out-of-hospital critical care medicine. Ann Emerg Med 34:738-44

24. Miner JR, Moore JC, Austad EJ, et al (2015) Randomized, double-blinded, clinical trial of propofol, 1:1 propofol/ketamine, and $4: 1$ propofol/ketamine for deep procedural sedation in the emergency department. Ann Emerg Med 65:479-88 